\title{
Combined channel estimation and PAPR reduction technique for MIMO-OFDM systems with null subcarriers
}

\author{
Emmanuel Manasseh*, Shuichi Ohno and Masayoshi Nakamoto
}

\begin{abstract}
In this article, we address the challenges regarding the provision of channel state information as well as reducing peak-to-average power ratio (PAPR) of a multiple input multiple output orthogonal frequency multiplexing (MIMO-OFDM) system. The mean squared error (MSE) of the channel estimate is adopted as the optimization criterion to design pilot symbols for channel estimation in MIMO-OFDM systems with null subcarriers. We design the placement and power distribution to the pilot symbols for multiple transmit antennas to minimize the MSE of the least square (LS) channel estimates. To reduce interference of the pilot symbols transmitted from different antennas, an algorithm to guarantee that pilot symbols are disjoint from any other transmitter pilot set is proposed. To efficiently reduce the PAPR of the MIMO-OFDM signals, a method that mixes dummy symbols and phase information of the pilot symbols is presented. Simulation results based on IEEE 802.16e are presented to illustrate the superior performance of our proposed channel estimation method over the existing standard and the partially equi-spaced pilot symbols. We also demonstrate that, by mixing the dummy symbols and phase information of the pilot symbols, the PAPR of the MIMO-OFDM signals can significantly be reduced.
\end{abstract}

Keywords: MIMO-OFDM, Dummy symbols, Mean square error (MSE), Peak-to-average power ratio (PAPR)

\section{Introduction}

Robustness of orthogonal frequency multiplexing (OFDM) systems in multipath environments together with the significant information capacity gain as well as improved bit error rate (BER) performance of multiple input multiple output (MIMO) systems, highlight the substantial potential of MIMO-OFDM systems [1-3]. However, in comparison to a single input single output (SISO) system with only one channel to be estimated, a MIMO system with $N_{t}$ transmit and $N_{r}$ receive antennas necessitates $N_{t} \times N_{r}$ channels to be estimated. This increased number of channels to be estimated may reduce the higher data rate of a MIMO system if pilot subcarriers are not well optimized [1].

In addition, OFDM signals exhibit high peak-to-average power ratio (PAPR), causing MIMO-OFDM signals

*Correspondence: manassehjc@hiroshima-u.ac.jp

Department of Artificial Complex Systems Engineering, Hiroshima University, 1-4-1 Kagamiyama Higashi-Hiroshima, 739-8527, Japan transmitted from different antennas to exhibit a prohibitively PAPR [4]. High PAPR is a critical issue in any multi-carrier system using OFDM. It can result in low power efficiency and large performance degradation of the system due to the nonlinearity of high-power amplifier $[5,6]$. Thus, it is desirable for the pilot symbols primarily designed for channel estimation to have low PAPR.

In the literature, training signal design for channel estimation have predominantly been developed for SISO-OFDM systems (see [7-13], and the reference therein). Optimal pilot symbols for OFDM systems in the absence of null edge subcarriers are considered in [7-10] where equi-distant and equi-powered pilot symbols were found to be optimal with respect to several performance measures.

In $[11,14]$, an algorithm for optimal preamble and pilot symbols design for SISO-OFDM systems with null subcarriers is considered. Both pilot power and placement are obtained by minimizing the mean squared error (MSE) of a channel estimate with convex optimization methods. The same problem is addressed in [12] where

\section{Springer}

(c) 2012 Manasseh et al.: licensee Springer. This is an Open Access article distributed under the terms of the Creative Commons Attribution License (http://creativecommons.org/licenses/by/2.0), which permits unrestricted use, distribution, and reproduction in any medium, provided the original work is properly cited. 
the placement of pilot symbols is obtained by parametric optimization, while the pilot power is obtained by minimizing the infinite norm of the channel MSE with convex optimization. Also in [13], a scheme that uses fifth-order parameterizations to obtain optimal pilot placement together with the analytical solution for the pilot power distribution is presented.

A number of pilot symbols design methods for MIMOOFDM systems have been studied as well, e.g., in [2,3,15-17]. In [16], equi-powered pilot symbols are studied for channel estimation in multiple antenna OFDM system with null subcarriers. But, they are not always optimal even for point-to-point OFDM system. In [3], partially equi-spaced pilot symbols (PEP) for MIMO-OFDM with null edge subcarriers is proposed. The algorithm in [3] is effective as it can be used to design pilot symbols for MIMO-OFDM systems with different frame sizes. Furthermore, the design considers both the placement and power distribution to the pilot symbols. However, the method does not guarantee better performance for some channel/subcarriers configuration.

In this article, we utilize the method proposed in $[11,14]$ for SISO systems where pilot symbols are obtained from the optimal preamble by iterative removal of subcarriers with minimum power. We extend this technique to MIMO systems with some modifications to ensure that pilot symbols of one antenna are disjoint from the pilot symbols of any other antenna. A modified algorithm is proposed to ensure that the composite pilot sequence from all antennas are positioned in the active subcarriers and are placed symmetrically about the center of the active subcarrier zone. The approach introduces a new pilot design paradigm that supports a prominent number of transmit antennas with more tractability in terms of complexity as well as applicability to OFDM systems with different channel/subcarriers configurations.

To reduce the PAPR of the MIMO-OFDM signals, a method that mixes tone reservation (TR) technique and phase information of the pilot symbols is presented. First, we utilize cross entropy (CE) optimization techniques to design phase information of the pilot symbols dedicated for channel estimation. Then, we employ convex optimization techniques to design dummy symbols (i.e., symbols not conveying information) to a reserved set of subcarriers to significantly reduce the PAPR of the transmitted data in an OFDM symbol.

Several design examples based on IEEE 802.16e are provided in Section IV, to demonstrate the efficacy of our proposed design.

The rest of this article is organized as follows: The MIMO-OFDM system model is briefly described in Section II. Channel estimation in MIMO-OFDM is concisely presented in Section III, while the proposed multiple antennas pilot design is addressed in Section IV. In
Section V, we illustrate the techniques employed to mitigate the PAPR problem. Simulation results demonstrating the effectiveness of our PAPR reduction techniques together with the performance of our proposed channel estimation algorithm as compared to the standard and the PEP schemes [3] are presented in Section VI. Finally, Section VII concludes this article.

\section{MIMO-OFDM system model}

A frequency selective MIMO-OFDM wireless system with $N_{t}$ transmit and $N_{r}$ receive antennas is considered. We assume that the discrete-time baseband equivalent channel between each transmit-receive antenna link has frequency impulse response of maximum length $L$, and remains constant in at least one OFDM symbol, i.e., quasistatic. Let us denote the channel from the $i$ th transmit antenna to the $m$ th receive antenna as

$$
\boldsymbol{h}_{\text {im }}=\left[h_{\text {im }}[0], h_{\text {im }}[1], \ldots, h_{\text {im }}[L-1]\right]^{T} \text {. }
$$

Our OFDM symbol is assumed to have $N$ subcarriers. We consider one OFDM symbol duration and denote the transmitted OFDM symbol from the $i$ th transmit antenna as

$$
\boldsymbol{X}_{i}=\left[X_{i}[0], X_{i}[1], \ldots, X_{i}[N-1]\right]^{T} .
$$

At the transmitter, each $X_{i}$ undergoes serial-to-parallel (S/P) conversion followed by an $N$-points inverse discrete Fourier transform (IDFT) to produce an OFDM symbol. Each OFDM symbol is parallel-to-serial (P/S) converted and a cyclic prefix $(\mathrm{CP})$ of length $N_{\mathrm{cp}}$ is appended to mitigate the multipath effects. Then, our discrete-time baseband equivalent transmitted signals can be expressed as

$$
x_{i}[n]=\frac{1}{\sqrt{N}} \sum_{k=0}^{N-1} X_{i}[k] e^{j \frac{2 \pi k n}{N}}, \quad n \in[0, N-1] .
$$

Assume that $N_{\mathrm{cp}} \geq L$ so that there is no inter-symbol interference (ISI) between consecutive OFDM symbols. At the receiver, we assume perfect timing synchronization.

After removing CP and perform DFT operations, the received frequency-domain signal at the $m$ th receive antenna is given by

$$
Y_{m}[k]=\sum_{i=1}^{N_{t}} H_{i m}[k] X_{i}[k]+W_{m}[k],
$$

where $H_{i m}[k]$ is the channel frequency response of the $(i, m)$ th channel at frequency $2 \pi k / N$ given by

$$
H_{i m}[k]=\sum_{l=0}^{L-1} h_{i m}[l] e^{-j \frac{2 \pi k l}{N}},
$$

and $\left\{W_{m}[k]\right\}$ is the DFT of the white Gaussian noise with variance $\sigma_{w}^{2}$. 


\section{Channel estimation in MIMO-OFDM}

For a discrete set $\mathcal{I}$, we denote $|\mathcal{I}|$ as the cardinality of $\mathcal{I}$. Let $\mathcal{K}_{s}$ be a set of active subcarriers. We assume the number of pilot symbols in each OFDM symbol to be $N_{p}$. For an OFDM symbol transmitted from the $i$ th transmit antenna, we place pilot and data symbols at subcarrier sets denoted as $\mathcal{K}_{p_{i}}$ and $\mathcal{K}_{d_{i}}$, respectively. To avoid interference between pilot symbols from different antennas, we set $\mathcal{K}_{p_{i}}$ for $i=1,2, \ldots, N_{t}$ to be disjoint, hence

$$
\mathcal{K}_{p_{i}} \cap \mathcal{K}_{p_{n}}=\emptyset \text { for } i \neq n .
$$

We also assume that there are no pilot symbols at $\mathcal{K}_{d_{i}}$, that is,

$$
\mathcal{K}_{d_{i}} \subseteq \mathcal{K}_{s} \backslash\left(\mathcal{K}_{p_{1}} \cup \mathcal{K}_{p_{2}} \ldots \cup \mathcal{K}_{p_{N_{t}}}\right)
$$

where $\backslash$ denotes set difference. Equation (7) implies that all transmit antennas utilize the same set of subcarriers for carrying data. Let us denote the positions of pilots symbols as

$$
\mathcal{K}_{p_{i}}=\left\{k_{i, 1} \ldots, k_{i, N_{p}}\right\} \text {. }
$$

Note that for each antenna transmitting an OFDM symbol with $\mathcal{K}_{s}$ active subcarriers, only $K_{p_{i}}$ and $\mathcal{K}_{d_{i}}$ are used for pilot and data transmission, while subcarriers corresponding to the pilot symbols of other transmit antennas are nulled. Thus, to transmit data symbols, it is necessary to satisfy the condition $\left|\mathcal{K}_{s}\right|-N_{p} N_{t}>0$. For each transmit antenna link there are $N_{v}=\left|K_{p} \backslash K_{p_{i}}\right|$ number of unused subcarriers within the active subcarrier band.

Since the same channel estimation process is performed at each receive antenna, we only need to consider a system with $N_{t}$ transmit antennas and one receive antenna in designing pilot symbols, that is, the channel is modeled as a superposition of multiple-input single-output (MISO) channels, as in $[3,17]$. Thus, without loss of generality, we can describe the first receive antenna and omit the index $m$ of the receive antenna.

Suppose that we estimate channels for coherent detection with pilot sets $\mathcal{K}_{p_{1}}, \mathcal{K}_{p_{2}}, \ldots, \mathcal{K}_{p_{N_{t}}}$. Let us define the frequency-domain channel gain as

$$
\boldsymbol{H}_{i}=\left[H_{i}\left[k_{1}\right], \ldots, H_{i}\left[k_{\left|\mathcal{K}_{s}\right|}\right]\right]^{T},
$$

where $k_{n}<k_{n^{\prime}}$ if $n<n^{\prime}$.

We define $\boldsymbol{F}$ as an $N \times N$ DFT matrix whose $(k+1, n+$ 1)th entry is $e^{-j 2 \pi k n / N}$, and

$$
\boldsymbol{F}_{L}=\left[\boldsymbol{f}_{0}, \ldots, \boldsymbol{f}_{N-1}\right]^{\mathcal{H}}
$$

as an $N \times L$ matrix consisting of $N$ rows and the first $L$ columns of a DFT matrix $\boldsymbol{F}$, where (.) ${ }^{\mathcal{H}}$ is the complex conjugate transpose operator. We also define an $N_{p} \times L$ matrix $\boldsymbol{F}_{p_{i}}$ having $\boldsymbol{f}_{k_{i, n}}^{\mathcal{H}}$ for $k_{i, n} \in \mathcal{K}_{p_{i}}$ as its $n$th row.
Then, the received signals in (4) having pilot symbols from the $i$ th transmit antenna is expressed as

$$
\boldsymbol{Y}_{i}=\boldsymbol{D}_{p_{i}} \boldsymbol{F}_{p_{i}} \boldsymbol{h}_{i}+\boldsymbol{W}_{i}
$$

where $\boldsymbol{D}_{p_{i}}$ is a diagonal matrix constructed from pilot symbols in the OFDM symbol transmitted from the $i$ th transmit antenna and $W_{i}$ is the corresponding sub-vector of $W_{m}$.

Similar to $\boldsymbol{F}_{p_{i}}$, we define a $\left|\mathcal{K}_{s}\right| \times L$ matrix $\boldsymbol{F}_{s}$ having $\boldsymbol{f}_{k_{n}}^{\mathcal{H}}$ for $k_{n} \in \mathcal{K}_{s}$ as its $n$th row, where $k_{n}<k_{n^{\prime}}$ if $n<n^{\prime}$. Then, we obtain

$$
\boldsymbol{H}_{i}=\boldsymbol{F}_{s} \boldsymbol{h}_{i} .
$$

From (11) and (12), the LS estimate $\hat{\boldsymbol{H}}_{i}$ of $\boldsymbol{H}_{i}$ is given by

$$
\hat{\boldsymbol{H}}_{i}=\boldsymbol{F}_{S}\left(\boldsymbol{F}_{p_{i}}^{\mathcal{H}} \boldsymbol{\Lambda}_{p_{i}} \boldsymbol{F}_{p_{i}}\right)^{-1}\left(\boldsymbol{D}_{p_{i}} \boldsymbol{F}_{p_{i}}\right)^{\mathcal{H}} \boldsymbol{Y}_{i},
$$

where

$$
\boldsymbol{\Lambda}_{p_{i}}=\boldsymbol{D}_{p_{i}}^{\mathcal{H}} \boldsymbol{D}_{p_{i}}=\operatorname{diag}\left(\lambda_{i, 1}, \ldots, \lambda_{i, N_{p}}\right) .
$$

Let us define the sum of the mean squared error (MSE) of the channel gain at $\mathcal{K}_{s}$ as

$$
\eta_{i}=E\left\{\left\|\hat{\boldsymbol{H}}_{i}-\boldsymbol{H}_{i}\right\|^{2}\right\},
$$

where $\|\cdot\|$ is the Euclidean norm, i.e., $\ell_{2}$ norm. Then, the channel MSE $\eta_{i}$ can be expressed as [11-14]

$$
\eta_{i}=\boldsymbol{F}_{s}\left[\frac{1}{\sigma_{w}^{2}}\left(\boldsymbol{F}_{p_{i}}^{\mathcal{H}} \boldsymbol{\Lambda}_{p_{i}} \boldsymbol{F}_{p_{i}}\right)\right]^{-1} \boldsymbol{F}_{s}^{\mathcal{H}} .
$$

For a given pilot set, the optimal pilot power $\lambda_{i, 1}, \ldots, \lambda_{i, N_{p}}$ that minimizes the channel MSE $\eta_{i}$ can be found numerically by resorting to convex optimization technique [11].

Since we have $N_{t}$ transmit antennas, the average MSE of the LS channel estimate for each receive antenna is given by

$$
\xi=\frac{\sigma_{w}^{2}}{N_{t}} \sum_{i=1}^{N_{t}} \operatorname{tr}\left[\boldsymbol{F}_{s}\left(\boldsymbol{F}_{p_{i}}^{\mathcal{H}} \boldsymbol{\Lambda}_{p_{i}} \boldsymbol{F}_{p_{i}}\right)^{-1} \boldsymbol{F}_{s}^{\mathcal{H}}\right]
$$

Note that, to design pilot symbols for spatially correlated MIMO-OFDM channels requires prior information of the channel which is not available in practical systems. We consider the general case and utilize the least square (LS) estimator to design pilot symbols.

In the following, based on (17), we determine the sets $\mathcal{K}_{p_{1}}, \mathcal{K}_{p_{2}}, \ldots, \mathcal{K}_{p_{N_{t}}}$ and power distributions to pilot subcarriers by using convex optimization techniques.

\section{Pilot design for MIMO-OFDM}

In our proposed design, we consider a MIMO scenario where disjoint pilot symbols are utilized for each transmit antenna. That is, if a pilot is allocated at the $k$ th subcarrier for one antenna no other antenna allocates a pilot or data in this subcarrier. Hence, inter-antenna interference can be avoided due to the null subcarrier assigned by other 
transmit antennas. Pilots can be inserted in any locations in the active band which match this criterion. For symbol by symbol channel estimation, disjoint set have been widely studied in the literature (see $[2,3,16,18]$ and the references therein).

This philosophy could be also employed to a scenario with any number of transmit antennas, calling for more null subcarriers for each additional transmit antenna. There is a spectrum wastage especially when large number transmit antenna is to be used. However, for MIMO systems where channel and other impairments are to be estimated independently for each OFDM symbol (i.e., symbol-by-symbol estimation), the disjoint pilot set is one of the prominent candidate. Another scheme that avoid the spectrum wastage is the phase shifted pilot symbols where the power distribution and placement of the pilot symbols is the same for all transmit antennas. In the phase shifted pilot design, only phase of the pilot symbols are different from each antenna. However, in reference [3] it is clearly demonstrated that the well designed disjoint pilot symbols outperform the phase shifted pilot symbols. Thus, for systems that utilizes a few transmit antennas eg. $N_{t}=2$, or for systems with large number of subcarriers the disjoint set is a prominent candidate.

\section{Pilot placement and power distribution}

To determine pilot sets and power distributions to the pilot subcarriers, we modify the algorithm in [11] to accommodate multiple antennas while guaranteeing that the designed pilot sets are disjoint from each transmit antenna. The main objective of disjoint pilot sequences in each transmit antenna is to ensure appropriate separation of pilot sequences at the receiver.

Pilot set for the first transmit antenna is obtained from the designed optimal preamble with semidefinite programming (SDP) by iterative removal of a certain number $N_{m}$, of subcarriers with minimum power symmetrically about the active subcarrier set, followed by optimization of the remaining subcarriers as in $[11,14]$.

Once the pilot set for the first transmit antenna is found, it is excluded from the active subcarrier set and pilot set for the second transmit antenna is obtained from the remaining active subcarriers by repeating the iterative algorithm. The algorithm is executed until pilot sets for all $N_{t}$ transmit antennas are obtained.

The modified placement and power design procedure for $N_{t}$ transmit antennas is summarized as follows:

1. Initialize $\mathcal{K}_{r}=\mathcal{K}_{s}$, where $\mathcal{K}_{r}$ stands for the set of available subcarriers.

2. while $i=1, \ldots, N_{t}$

(a) Define the temporary set $\mathcal{K}_{t}=\mathcal{K}_{r}$ and optimize $\mathcal{K}_{t}$ subcarriers using convex optimization (b) Save the obtained position and power of the subcarriers

(c) If $N_{p}<\left|\mathcal{K}_{t}\right|$, remove $N_{m}$ subcarriers with minimum power symmetrically with respect to the central $D C$ subcarrier, else go to step $\mathrm{f}$ )

(d) Update $\mathcal{K}_{t}\left(\left|\mathcal{K}_{t}\right|=\left|\mathcal{K}_{t}\right|-N_{m}\right)$.

(e) Optimize the power of the remaining subcarriers using SDP and go to step b)

(f) Save the pilot position as $\mathcal{K}_{p_{i}}$ and its power distribution

(g) Update $\mathcal{K}_{r}=\mathcal{K}_{r} \backslash \mathcal{K}_{p_{i}}, i \leftarrow i+1$ and return to step a) until $i \geq N_{t}$

In the algorithm, once the set $\mathcal{K}_{p_{i}}$ is obtained, it is excluded from the remaining active subcarriers $\mathcal{K}_{r} \backslash \mathcal{K}_{p_{i}}$. This assures the unique pilot placement of the optimized pilot set from each transmit antenna. That is, the locations of the pilot symbols for each transmit antenna are distinct from the pilot set of any other transmit antenna within the active subcarrier band. Note that the symmetrical removal of $N_{m}$ subcarriers after every optimization guarantee the symmetrical placement of pilot sets about the center of the signal band.

When the algorithm exits, we obtain the pilot positions and the normalized pilot powers for each antenna. To optimally distribute power between pilot symbols and data subcarriers, we can also modify the method in $[11,14]$ depending on the data transmission scheme. If one adopts OFDMA for data transmission, the method in [11] can directly be applied, while for space time block coding or other schemes, the method in [11] calls for some modifications.

It should noted that, for a given set of antennas, we did not consider the order intuitively, we simply considered the antenna index from 1 to $N_{t}$ and design pilot set for each antenna, one by one for all transmit antennas. Generally, the order of the optimization with respect to transmit antennas is not critical to the final performance. However, total number of active subcarriers $\left|\mathcal{K}_{s}\right|$, number of transmit antennas $N_{t}$, and pilot symbols $N_{p}$, may affect the final performance. For systems with large number of transmit antennas, it is likely possible for the pilot symbols of the first designed antenna to have better performance than the last one. In such situation, to obtain better performance, pilot rearrangement between transmit antennas or some other existing selection algorithms can be utilized.

The proposed convex optimization design can be modified and be applied in the systems where the prior information of the channel is available. In [19], it has been shown that the optimization problem is still convex when spatial channels are considered. This suggests that, even though the scheme in [19] is different from the proposed 
method as it design training or preamble symbols for multiple transmitted OFDM symbols, the proposed design may also be adopted in spatially correlated channels.

\section{PAPR in MIMO-OFDM signals}

For a given transmitter-receiver link with $N$ subcarriers in one OFDM symbol, let

$$
X_{i}=\left[X_{i}[0], X_{i}[1], \ldots, X_{i}[N-1]\right]^{T}
$$

be the amplitudes of $N$ subcarriers from the $i$ th transmit antenna. At the transmitter, each $X_{i}$ undergoes $\mathcal{L} N$-points IDFT to produce the $\mathcal{L}$-times oversampled-time domain baseband signals expressed as

$$
\boldsymbol{x}_{i}=\boldsymbol{\Gamma} \boldsymbol{X}_{i}
$$

where $\boldsymbol{\Gamma}$ is an $\mathcal{L} N \times N$ DFT matrix with

$$
\begin{aligned}
\Gamma_{t, k}=\frac{1}{\sqrt{\mathcal{L} N}} e^{j \frac{2 \pi k t}{\mathcal{L} N},} \quad t & \in[0, \mathcal{L} N-1], \\
k & \in[0, N-1] .
\end{aligned}
$$

Note that, $\mathcal{L}$ denotes the oversampling factor sufficient to make the signal $\boldsymbol{x}_{\boldsymbol{i}}$ as close as possible to the continuous signal [5]. The average power of the signal is defined as

$$
E\left\{\left|\boldsymbol{x}_{i}\right|^{2}\right\}=\frac{1}{\mathcal{L} N} \int_{0}^{\mathcal{L} N}\left|x_{i}(t)\right|^{2} d t=\sum_{n=0}^{N-1}\left|X_{i}[n]\right|^{2},
$$

where $E\{\cdot\}$ denotes expectation operation. The peak-toaverage power (PAPR) of the transmitted signal in (19) is defined as

$$
P A P R_{i}=\frac{\left\|\boldsymbol{x}_{i}\right\|_{\infty}^{2}}{E\left\{\left|\boldsymbol{x}_{i}\right|^{2}\right\}},
$$

where $\left\|\boldsymbol{x}_{i}\right\|_{\infty}$ is the infinity norm of the time domain signals. The above definition clarifies that the PAPR is the maximum instantaneous power normalized by the average power among all possible signal patterns.

To avoid nonlinear distortion in the power amplifiers and in turn the generation of undesired out-of-band radiation, the PAPR of all $N_{t}$ transmit signals should be simultaneously as small as possible [20]. Since the performance is governed by the worstcase PAPR, we define $P A P R_{\mathrm{MIMO}}$ as the maximum of all PAPR related to all $N_{t}$ MIMO path $[4,20]$. Thus,

$$
P A P R_{M I M O}=\max _{i=1, \ldots, N_{t}} P A P R_{i} .
$$

Note that PAPR is a random variable and the suitable description is the complementary cumulative distribution function (CCDF), which gives the probability $v_{o}$ of exceeding a specified threshold $\gamma$, i.e.,

$$
v_{o}=\operatorname{Pr}[P A P R>\gamma] \text {. }
$$

In the following, we will demonstrate some techniques that can be used for PAPR reduction of OFDM signals.

\section{PAPR reduction using phase information of the pilot symbols and TR techniques}

For an OFDM symbol that consists of unused subcarriers, pilot and data subcarriers, phase information of the pilot tones together with a certain number of unused subcarriers can be utilized to mitigate the problem of high PAPR in OFDM systems. Careful design of the phase information to the pilot symbols can substantially minimize the peak levels of the time domain OFDM signals [21]. In line with the phase information of the pilot symbols, tone reservation (TR) technique which makes use of some reserved or unused subcarriers and insert dummy symbols that simultaneously minimize the peak levels of the sampled time domain OFDM signals can be utilized.

In this article, a method that mixes TR technique and phase information of the pilot symbols to efficiently reduce the PAPR of the MIMO-OFDM signals is presented. First, transmitters that reduce the PAPR by utilizing phase information of the pilot symbols is introduced. We adopt the techniques proposed in [21] to design phase information of the pilot symbols primarily dedicated for channel estimation (see Section IV and [3]) to reduce the PAPR of an OFDM symbol. Then, TR technique is employed to design dummy symbols that effectively reduce the PAPR. The optimal power distribution and phase information of the dummy symbols are determined by the solution of a convex optimization problem.

Let $\mathcal{K}_{v}$ be a set of dummy symbols for each transmit antenna link, then we denote the number of dummy symbols as $N_{v}=\left|\mathcal{K}_{v}\right|$. Suppose that, we define $\boldsymbol{\Gamma}_{\boldsymbol{d}}, \boldsymbol{\Gamma}_{\boldsymbol{p}_{i}}$ and $\boldsymbol{\Gamma}_{\boldsymbol{v}}$ as the DFT submatrix of $\boldsymbol{\Gamma}$ corresponding to $\mathcal{K}_{d}, \mathcal{K}_{p_{i}}$ and $\mathcal{K}_{\nu}$ subcarriers, respectively. Then, we can decompose the expression in (19) as

$$
\boldsymbol{x}_{i}=\boldsymbol{\Gamma}_{d} \boldsymbol{X}_{d_{i}}+\boldsymbol{\Gamma}_{p_{i}} \boldsymbol{X}_{p_{i}}+\boldsymbol{\Gamma}_{v} \boldsymbol{X}_{v_{i}}
$$

where $\boldsymbol{X}_{d_{i}}, \boldsymbol{X}_{p_{i}}$ and $\boldsymbol{X}_{v_{i}}$ are the vectors containing data, pilot, and dummy symbols, respectively. In the following sections, we will discuss these techniques used to counteract the PAPR problem.

\section{Pilot phase design for PAPR reduction}

For channel or carrier frequency offset estimation, it is sufficient to design the placement and power of each pilot symbol, and there are no particular requirements for the phase information. Thus, we can utilize phase information of the pilot symbols to enhance a reasonable PAPR reduction. We consider a set of frequency domain pilot symbols designed for channel estimation of the received OFDM symbol from the $i$ th transmit antenna and design plausible phase information that lower the peak amplitudes of the pilot symbols in time domain. 
From Equation (25), for a given set of pilot symbols in frequency domain, the corresponding time domain representation of the pilot symbols with phase information can be written as

$$
\boldsymbol{x}_{p_{i}}=\boldsymbol{\Gamma}_{p_{i}} \operatorname{diag}\left(\boldsymbol{X}_{p_{i}}\right) e^{j \phi_{i}}
$$

where $\boldsymbol{\phi}_{\boldsymbol{i}}$ is an $N_{p} \times 1$ vector containing phase information of the pilot symbols from the $i$ th transmit antenna link.

From (26), we can write the peak minimization problem as

$$
\min _{\phi_{i}}\left\|\boldsymbol{x}_{p_{i}}\right\|_{\infty}=\min _{\phi_{i}}\left\|\boldsymbol{\Gamma}_{p_{i}} \operatorname{diag}\left(\boldsymbol{X}_{p_{i}}\right) e^{j \phi_{i}}\right\|_{\infty}
$$

which is the minimization of the maximum amplitude of the time domain signals $\boldsymbol{x}_{p_{i}}$.

Phase design to reduce PAPR is a non-convex and nonlinear optimization problem [21]. The non-convex optimization problem is addressed in [22], where exhaustive search method is employed to design phase information of the pilot symbols. However, the exhaustive search scheme becomes computationally prohibitive especially for pilot sets with a large number of subcarriers. Furthermore, the performance of the scheme depends on the searching granularity. In [21], phase information of the pilot symbols are obtained by the CE optimization techniques. Compared to the exhaustive search method, the algorithm in [21] converges fast to the near optimal solution. Due to its high convergence rate, the scheme has a potential to make practical design of phases for different applications. Thus, we resort to the later approach and slightly modify it to be used for the design of pilot phase information when multiple transmit antennas are employed, while limiting the number of iterations without improvement.

\section{CE-based phase optimization techniques}

We utilize CE optimization method to design random phase information to a given set of pilot symbols to reduce the PAPR of the time domain pilot symbols. In most practical cases, the power levels loaded to the pilot symbols in frequency domain is relatively higher than the power loaded to the data subcarriers. Thus, by lowering the peak levels of the pilot symbols, the PAPR of the whole OFDM symbol can be slightly reduced.

The basic idea behind the CE method is to transform the original (combinatorial) optimization problem to an associated stochastic optimization problem, and then to tackle the stochastic problem efficiently by an adaptive sampling algorithm. By doing so, one constructs a random sequence of solutions which converges (probabilistically) to the optimal or at least a reasonable solution. Once the associated stochastic optimization is defined, the CE method alternates the following two phases:

1. Generation of a sample of random data according to a specified random mechanism.
2. Update of the parameters of the random mechanism, on the basis of the sample data, in order to produce a better sample in the next iteration.

From the problem formulated in (27), if we generate $M$ sample vectors of random phases, then the optimization problem can be expressed as

$$
\boldsymbol{\Psi}\left(\phi_{i}\right)=\min _{\phi_{i} \in \boldsymbol{\Phi}}\left\|\boldsymbol{x}_{p_{i}}\right\|_{\infty}=\min _{\phi_{i} \in \boldsymbol{\Phi}}\left\|\boldsymbol{\Gamma}_{p_{i}} \operatorname{diag}\left(\boldsymbol{X}_{p_{i}}\right) e^{j \phi_{i}}\right\|_{\infty} .
$$

Once the samples have been generated, the next step is to use the sample to modify the parameters of the random mechanism, in order to produce a better sample in the next iteration. The methodology is focusing on the observations of the best objective function values in order to bias the sampling process.

The proposed CE method employs $M$ number samples, the cutoff point for high-quality observations $\rho$, the smoothing constants $\alpha$ for updating the mean and standard deviation of the samples, the limit on the number of iterations without improvement $\mathcal{Z}$, the limit on the total number of iterations $\mathcal{J}$ and the limit on standard deviation $\epsilon$.

\section{Algorithm 1. CE algorithm}

1: Initialize $\hat{\mu}_{0}$ and $\hat{\sigma}_{0}$ and the CE parameters $\alpha, \beta$ and $\rho$.

2: Initialize $\boldsymbol{\Psi}\left(\phi^{*}\right)=\left\|\boldsymbol{x}_{p}^{0}\right\|_{\infty}$ and set the iteration counter $t=0$ and $t^{\prime}=0$.

3: while $\left(t^{\prime}<\mathcal{Z}\right.$ and $t<\mathcal{J}$ and $\left.\max _{k}\left(\hat{\sigma}_{k, t}\right)<\epsilon\right)$ \{

4: $\quad$ Generate random sample $\phi_{1}, \ldots, \phi_{M}$ from the distributions $\mathcal{N}\left(\hat{\mu}_{k, t-1}, \hat{\sigma}_{k, t-1}^{2}\right)$.

5: Compute $\boldsymbol{\Psi}(\phi)$ and order the sample in such a way that $\boldsymbol{\Psi}\left(\phi^{1}\right) \leq \boldsymbol{\Psi}\left(\phi^{2}\right) \ldots \leq \boldsymbol{\Psi}\left(\phi^{M}\right)$

6: Select $\mathcal{I}$ indices of the best performing samples, and calculate mean and standard deviation.

$\mu_{k, t}=\frac{1}{M_{\ell}} \sum_{i \in \mathcal{I}} \phi_{k, i}$ and

$\sigma_{k, t}^{2}=\frac{1}{M_{\ell}} \sum_{i \in \mathcal{I}}\left(\phi_{k, i}-\mu_{k, t}\right)^{2}$

7: $\quad$ Smoothen the mean and standard deviation of the best performing samples using

$$
\begin{aligned}
& \hat{\mu}_{k, t}=\alpha \mu_{k, t}+(1-\alpha) \hat{\mu}_{k, t-1} \text { and } \\
& \hat{\sigma}_{k, t}=\alpha \sigma_{k, t}+(1-\alpha) \hat{\sigma}_{k, n-1} \text { for } k=0, \ldots, N_{p}-1 . \\
8: & \text { if } \boldsymbol{\Psi}\left(\phi^{*}\right)<\boldsymbol{\Psi}\left(\phi^{1}\right) \text { then } \\
\text { 9: } & t^{\prime} \leftarrow t^{\prime}+1 \\
10: & \text { else } \\
11: & \boldsymbol{\Psi}\left(\phi^{*}\right)=\boldsymbol{\Psi}\left(\phi^{1}\right) \text { and } t^{\prime}=0 \\
12: & \text { end if } \\
13: & \text { Increment } t \leftarrow t+1
\end{aligned}
$$$$
\text { 10: else }
$$$$
\text { 12: end if }
$$$$
\text { 14: \} }
$$

The pseudocode of the CE method is as presented in Algorithm 5.2.1. The algorithm starts by initializing 
the mean and standard deviation as $\mu_{0}$ and $\sigma_{0}$, respectively. The best objective function value is initialized to $\left\|x_{p}^{0}\right\|_{\infty}$, which is the peak amplitude of the zero phase pilot symbols and the iteration counters $t$ (total number of iterations) and $t^{\prime}$ (iterations without improvement) are initialized to zero (see steps 1-2 in the pseudocode). The main loop includes the two main tasks that every CE method must perform, namely, the generation of a sample and the updating of the parameters associated with the chosen probability distribution. Step 4 generates $M$ sample vectors $\left\{\Phi_{m}\right\}_{m=1}^{M}$ of length $N_{p}$ using a family of normal probability density functions (PDF) $\mathcal{N}\left(\mu_{k}, \sigma_{k}^{2}\right)$ for $k=0, \ldots, N_{p}-1$.

The ordering of the sample in step 5 is such that the best observation is placed in the first position of the list and the worst is placed in the last position. The mean and standard deviation values calculated in step 6 corresponds to the variables of the top $M_{\ell}$ solutions in the current sample. Note that, a fixed number of the best performing samples $M_{\ell}$ are referred to as the elite samples expressed as $M_{\ell}=\rho M$. In step 7, we obtain the smoothed mean $\hat{\mu}_{k, t}$ and standard deviation $\hat{\sigma}_{k, t}$ by using some fixed smoothing parameter $\alpha$ where $0<\alpha<1$.

Steps 9 and 11 update the best solution found and reset the counter of the number of iterations without improvement, respectively. The global iteration counter is updated in step 13. Note that, the mean $\hat{\mu}_{k, t}$ converges to $\phi^{*}$ and the standard deviation $\hat{\sigma}_{k, t}$ to the zero. In brief, we obtain a degenerated PDF with all mass concentrated in the vicinity of the vector $\phi^{*}$.

At each stage $t$ of the CE procedure we simulate a sample $\phi$ from a $\mathcal{N}\left(\hat{\mu}_{t-1}, \hat{\sigma}_{t-1}^{2}\right)$ distribution, and update $\hat{\mu}_{t}$ and $\hat{\sigma}_{t}$ of the best samples $M_{\ell}$.

The pseudocode in Algorithm 5.2.1 is faster than the CE method proposed in [21], as it limits the number iterations without improvements. The algorithm can be utilized to design pilot phase information for all transmit antennas by repeating the same procedure for each set of pilot symbols

\section{TR for PAPR reduction}

TR technique is one of the promising approach for PAPR reduction in OFDM systems [5,23-25], where the transmitter mitigates the PAPR problem by sending dummy symbols (i.e., symbols not conveying information) in some reserved subcarriers [26]. The advantages of TR-based schemes is that there is no specific PAPR reduction information that needs to be communicated to the receiver. However, one problem with TR techniques lies on the computationally efficient determination of dummy symbols that effectively minimizes the PAPR [23]. The amount of PAPR reduction depends on some factors such as location of the dummy symbols, number of dummy symbols, and allowed power on these dummy symbols.
Some issues to be considered before using the TR techniques include PAPR reduction capacity, power increase in transmit signals and the loss in data rate. In the proposed TR technique, reduction in PAPR is achieved at the expense of increasing the total transmitted power of an OFDM symbol.

To reduce the PAPR we need to minimize $\left\|\boldsymbol{x}_{i}\right\|_{\infty}$, which is equivalent to the peak of the signal $\boldsymbol{x}_{i}$ in (25). The peak minimization problem can written as

$$
\min _{\boldsymbol{X}_{v_{i}}}\left\|\boldsymbol{x}_{i}\right\|_{\infty}=\min _{\boldsymbol{X}_{v_{i}}}\left\|\boldsymbol{\Gamma}_{d} \boldsymbol{X}_{d_{i}}+\boldsymbol{\Gamma}_{p_{i}} \boldsymbol{X}_{p_{i}}+\boldsymbol{\Gamma}_{v} \boldsymbol{X}_{v_{i}}\right\|_{\infty}
$$

Note that, for simplicity we consider that, a same set of null subcarriers is reserved for PAPR reduction on different transmit antenna link. However, power and phase information are different for each transmit antenna link, i.e., $\mathcal{K}_{v}=\mathcal{K}_{v_{1}}=\mathcal{K}_{v_{2}}=\ldots=\mathcal{K}_{v_{N_{t}}}$, and $\boldsymbol{X}_{v_{1}} \neq \boldsymbol{X}_{v_{2}} \neq$ $\ldots \neq X_{v_{N_{t}}}$.

For an OFDM symbol with $N_{G}$ number of null edge subcarriers or guard subcarriers, we need to select a set of $\mathcal{K}_{v}$ subcarriers and optimally allocate the power as well as phase information to ensure that the designed dummy symbols $\boldsymbol{X}_{v_{i}}$, significantly reduces the PAPR of each transmit antenna link.

\section{The placement of the dummy symbols}

In practice a considerable portion of subcarriers are reserved as guard subcarriers to avoid interferences from neighboring communication channels. In the proposed method, we utilize some of these guard subcarriers for PAPR reduction. The optimal placement can improve the efficiency of the dummy symbols in reducing PAPR. To obtain optimal location of dummy symbols for a fixed number of guard subcarriers $N_{G}$ and for a given number of dummy symbols $N_{v}$, the exhaustive method can be utilized [27]. In [27], it has been shown that scattered dummy symbol with maximum distance from adjacent dummy symbols outperforms the contiguous placements of the dummy symbols. However, exhaustive methods used to obtain optimal placements are not tractable for an OFDM symbols with large number of subcarriers. For example, in IEEE 802.16e standard an OFDM symbol consists 256 subcarriers, out of which 55 are nulled at the edges of the block. Suppose 16 subcarriers out of 55 are to be used for PAPR then there are $\left(\begin{array}{l}55 \\ 16\end{array}\right) \approx 2.97495 \times 10^{13}$ possible combinations. Furthermore, optimal placement of dummy symbols to minimize the associated PAPR of an OFDM symbol depends on various factors such as, the information loaded in the active subcarriers, power distribution to the dummy symbols and the phase information of these dummies. This calls for online update of the placement of these dummy symbols for each transmitted OFDM symbol which increases the complexity especially 
for a system with large number of guard subcarriers. Since there is no closed-form expression relating the placement of dummy symbols with the PAPR, we propose a simple symmetrical placement of the dummy symbols with maximum distance from adjacent dummy symbols. Then, we introduce separate algorithms for power loading and phase information.

The maximum distance between the adjacent dummy symbols is given by

$$
d=\left\lfloor\frac{N_{G}}{N_{v}-1}\right\rfloor
$$

where $\lfloor c\rfloor$ rounds $c$ to the nearest integer less than or equal to c. The dummy symbols are located at $\left[\frac{N_{G}-(2 \boldsymbol{a}-1) d}{2}, N-\right.$ $\left.\frac{N_{G}-(2 \boldsymbol{a}-1) d}{2}\right]$, where $\boldsymbol{a}=\left[\frac{N_{v}}{2}, \frac{N_{v}}{2}-1, \ldots 1\right]$. For $N_{v}=16$, dummy symbols are located at $\{5,8,11,14,17,20,23,26,230,233,245,247,251\}$.

Note that, it is also possible to start from the edges by placing the dummy symbols on right and left ends of the guard band subcarriers. In the following subsection we will introduce methods that can be used to load power and phase to the dummy subcarriers.

\section{Convex optimization-based TR techniques}

The main differences in the TR techniques are based on the selection of the (convex) cost function, the possible constraints set and the algorithms used to obtain an optimal solution. In [26], TR techniques that use adaptive projected subgradient method to obtain dummy symbols to minimize PAPR of each symbol is proposed, while in [28], a subgradient optimization-based framework for iterative PAPR reduction is proposed. Both [26] and [28] utilize iterative algorithm to obtain the peak canceling symbols. The accuracy of the iterative methods depends on the number of iterations and the selection of the updating parameters. The approach in [28] minimizes the peak magnitude of the OFDM symbol vector by using tone values of the reserved subcarriers which are iteratively updated through a subgradient search. The algorithms have very simple update rules and low computational complexities. However, the number of updates required for a satisfactory peak level tends to be high. Convex optimization techniques for PAPR reduction are also addressed in [29] and the references therein.

In our proposed TR techniques we employ convex optimization packages (cvx) in [30] to efficiently design dummy symbols (or peak canceling symbols) under certain constraints without employing iteration methods.

From the objective function in (29), it is desired to optimize the dummy symbols $\boldsymbol{X}_{v_{i}}$ to reduce the peaks of the time domain signal $\boldsymbol{x}_{i}$ to the lowest possible amplitude level. By combining pilot phase information in (26), our objective function can be expressed as

$$
\Omega\left(\boldsymbol{X}_{v_{i}}\right)=\min _{\boldsymbol{X}_{v_{i}}}\left\|\boldsymbol{\Gamma}_{d} \boldsymbol{X}_{d_{i}}+\boldsymbol{\Gamma}_{p_{i}} \operatorname{diag}\left(\boldsymbol{X}_{p_{i}}\right) e^{j \phi_{i}}+\boldsymbol{\Gamma}_{v} \boldsymbol{X}_{v_{i}}\right\|_{\infty} .
$$

Only $\boldsymbol{X}_{v_{i}}$ is allowed to change, thus we need to introduce some constraints to describe the desired characteristics of the convex set $\boldsymbol{X}_{v_{i}}$ and the signal amplitude constraints to limit the PAPR to an acceptable level.

Practically, one cannot select arbitrary values for $\boldsymbol{X}_{v_{i}}$, since they should obey the power spectral density (PSD) constraints for different applications imposed by the standards for the spectral compatibility reasons (see $[5,26,28]$ ). Therefore, the average power levels of the reserved tones are constrained by the PSD mask levels. Apart from the PSD constraints in frequency domain, we can add the signal peak level reduction requirement in time domain as well. Thus, we can write the peak minimization problem as the constrained optimization problem

$$
\begin{array}{cl}
\underset{\boldsymbol{X}_{v_{i}}}{\operatorname{minimize}} & \Omega\left(\boldsymbol{X}_{v_{i}}\right) \\
\text { subject to } & \left|X_{v_{i}}\right| \leq \chi, \\
& \Omega\left(\boldsymbol{X}_{v_{i}}\right) \leq \beta\left\|\boldsymbol{\Gamma}_{d} \boldsymbol{X}_{d_{i}}\right\|_{\infty}
\end{array}
$$

where $\chi$ is the PSD mask level constraint in frequency domain and $\beta$ is a fraction value representing the target peak level to be attained. From the constraints above, the phase of the dummy symbols can either be $0^{\circ}$ or $180^{\circ}$.

The convex optimization problem in (32) can efficiently be solved by the using the cvx optimization package in [30]. Note that, TR approach does not require any modifications at the receiver, thus, the receiver easily recover the transmitted data symbols by discarding subcarriers loaded with dummy symbols.

\section{Simulation results}

In this section, we demonstrate the effectiveness of our proposed pilot design through computer simulations. We set the noise variance $\sigma_{w}^{2}=1$. The parameters of the transmitted OFDM symbol studied in our design examples are similar to the IEEE 802.16e standard in [31, p. 429], where an OFDM transmission frame with $N=256$ is considered. Out of 256 subcarriers, 200 are data carrying subcarriers used for data and pilots. Of the remaining 56 subcarriers, 28 are null in the lower frequency guard band while 27 are nulled in the upper frequency guard band and one is the central DC null subcarrier. Of the 200 active subcarriers, 8 are allocated as pilot subcarriers, while the remaining 192 are used for data transmission.

\section{Pilot symbol design}

The minimum number of pilot symbols needed per OFDM symbol depend on the channel length, $L$ (see 
$[1,3,8,12]$ and the references therein). For the multipath channel to be estimated, the number of pilot symbols need to be at least equal to the length of the channel impulse response, i.e., $N_{p} \geq L$. The general assumption is that, taps of the channel impulse response beyond $L$ samples are insignificant [8], which is similar to the assumption made in OFDM to justify that no ISI occurs. Thus in our simulations we use $N_{p}=L$.

To design disjoint pilot symbols to multiple transmit antennas, we construct a composite pilot sequence with index sets $\left\{\mathcal{K}_{p_{i}}\right\}$ having $N_{t} N_{p}$ subcarriers with significant pilot power and reasonable position. The pilot set for the first antenna is obtained as in $[11,14]$, then by utilizing our proposed algorithm in Section IV, which exclude the designed pilot set from the preamble and repeat the same procedure for the remaining subcarriers to obtain pilot sets for all $N_{t}$ transmit antennas.

In the proposed design, total power allocated to the pilot symbols of each transmit antenna link is normalized to 1 . By normalizing the power of pilot symbols, one can easily distribute the power when total power dedicated to the pilot symbols is given.

The analytical solution for optimal pilot power distribution in [13] can also be adopted, for a given set of arbitrary pilot placement, [13] is a prominent candidate for OFDM systems with large number of subcarriers. In [13] to obtain the placement of the pilot symbols, fifthorder parameterization is employed. However, the fifthorder parameterization like the cubic parameterization in [12] requires contiguous subcarriers except for the central DC subcarriers. This limits the adoption of [12,13] in designing disjoint pilot symbols for OFDM systems with multiple transmit antennas.
Figure 1 shows the designed disjoint pilot set for four transmit antennas when $N_{p}=8$. For all transmit antennas, pilot power and location are well distributed within the in-band region which promises better estimation of the channels even at the edge of the band.

Figure 2 compares our proposed pilot symbols and the partially equi-spaced pilot (PEP) symbols proposed in [3] for $N_{p}=16$. In the two designs, total power dedicated to the pilot symbols is the same. For our proposed design power allocated to the edge pilot symbols is slightly lower than that of the mid pilot symbols, however the difference is not as large as in the PEP design. In [3], it is stated that, the power of pilot symbols decreases when the pilot symbols are close to the null/virtual carriers zone due to the fact that there are less data carriers, this might be true, however the power allocated to these subcarriers need to be significant, otherwise the problem of channel estimation via the extrapolation of the edge subcarriers will still persist [32].

\section{Comparison of MSE performance}

We evaluate the MSE performance of the designed pilots symbols with respect to the existing designs. First, we compare each of the designed pilot set with the existing IEEE 802.16e standard pilot symbols separately, i.e., SISO-OFDM mode. The aim is to observe the performance of the designed pilot symbols in each antenna with respect to the standard one to ensure that each designed pilot set has better performance. A noteworthy fact is that, when some SISO-OFDM methods are adopted in MIMO-OFDM pilot designs the performance of some designed pilot set deteriorates with increased number of transmit antennas. This

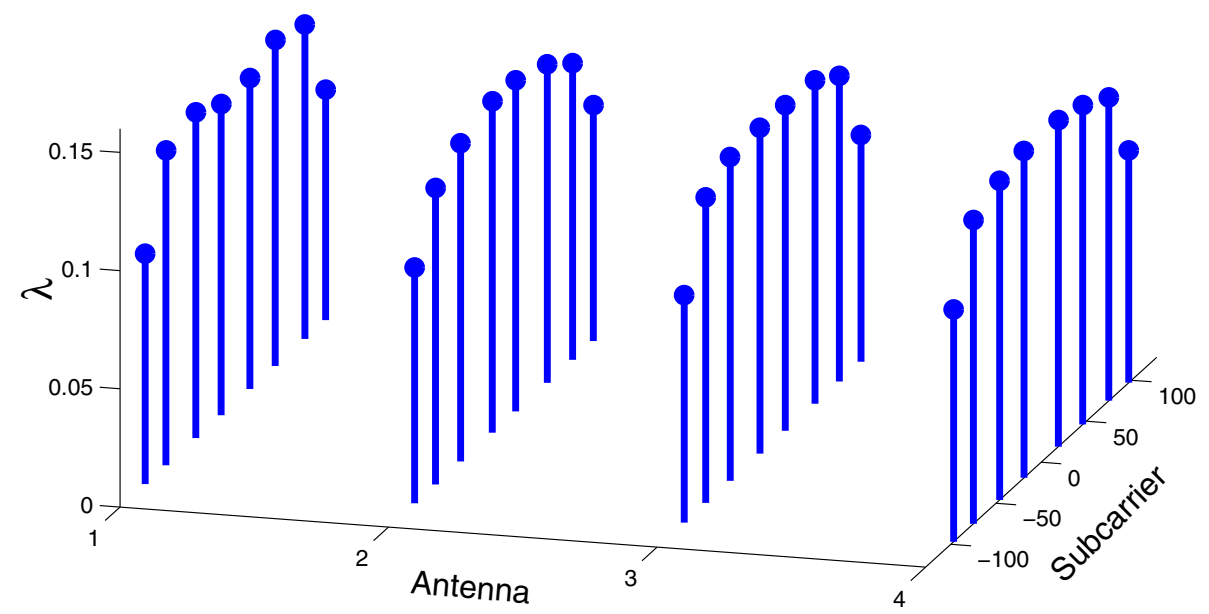

Figure 1 Pilot position and power distribution for four transmit antennas. 


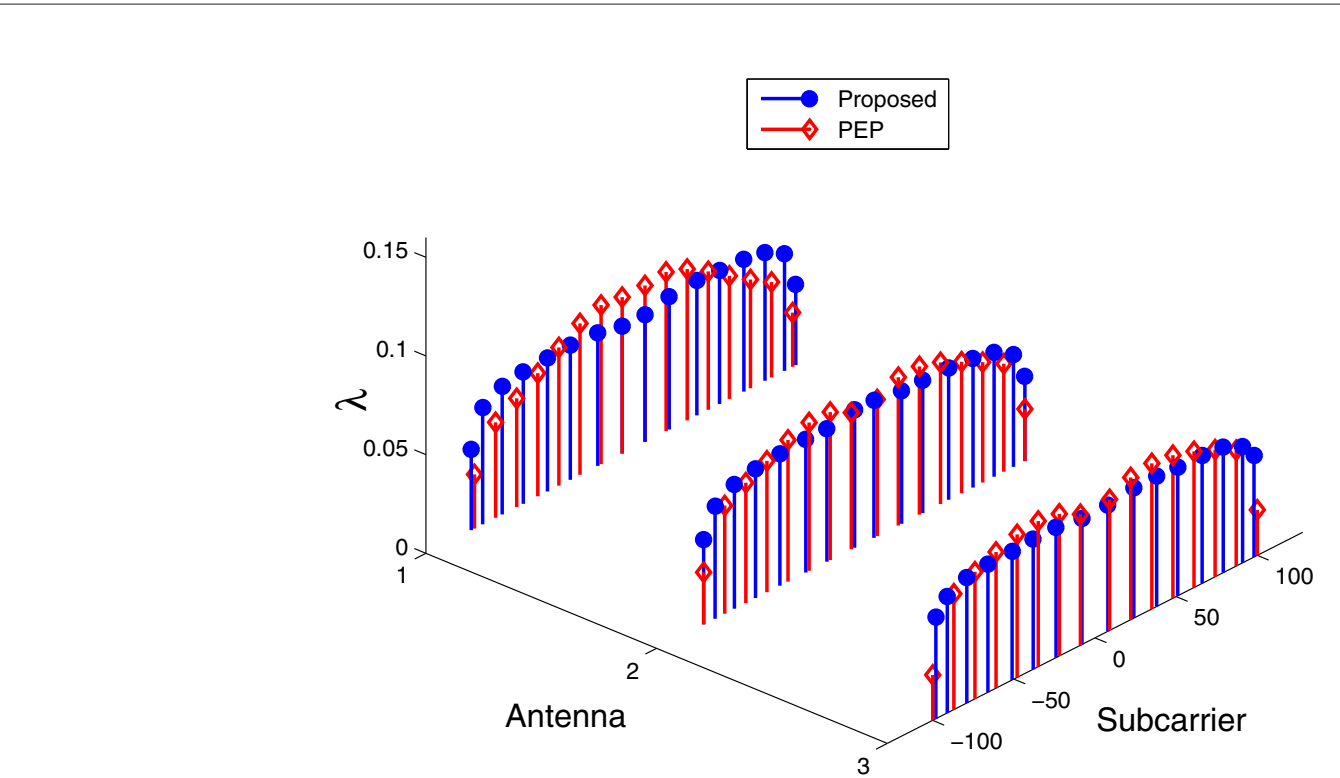

Figure 2 Comparison of pilot design for three transmit antennas.

implies that, only a few pilot sets yield a significant performance.

In Figure 3, the normalized channel estimate MSE of the designed disjoint pilot symbols in Figure 1 is compared with the existing standard which places the eight subcarriers at $\{ \pm 13, \pm 38, \pm 63, \pm 88\}$. The total pilot power for each antenna is taken to be $N_{p}$, for both the standard (equi-spaced, equi-powered pilot symbols) and our proposed method. From the plot it is clear that each individual antenna outperforms the conventional standard design. The standard pilot design does a poor job of estimating channel at the subcarriers near the guard band, due to lack of the pilot subcarriers at the edge of the OFDM symbol in IEEE 802.16e standard pilots. As a result, the estimation via extrapolation for the edge subcarriers results in a higher error [15]. A possible solution

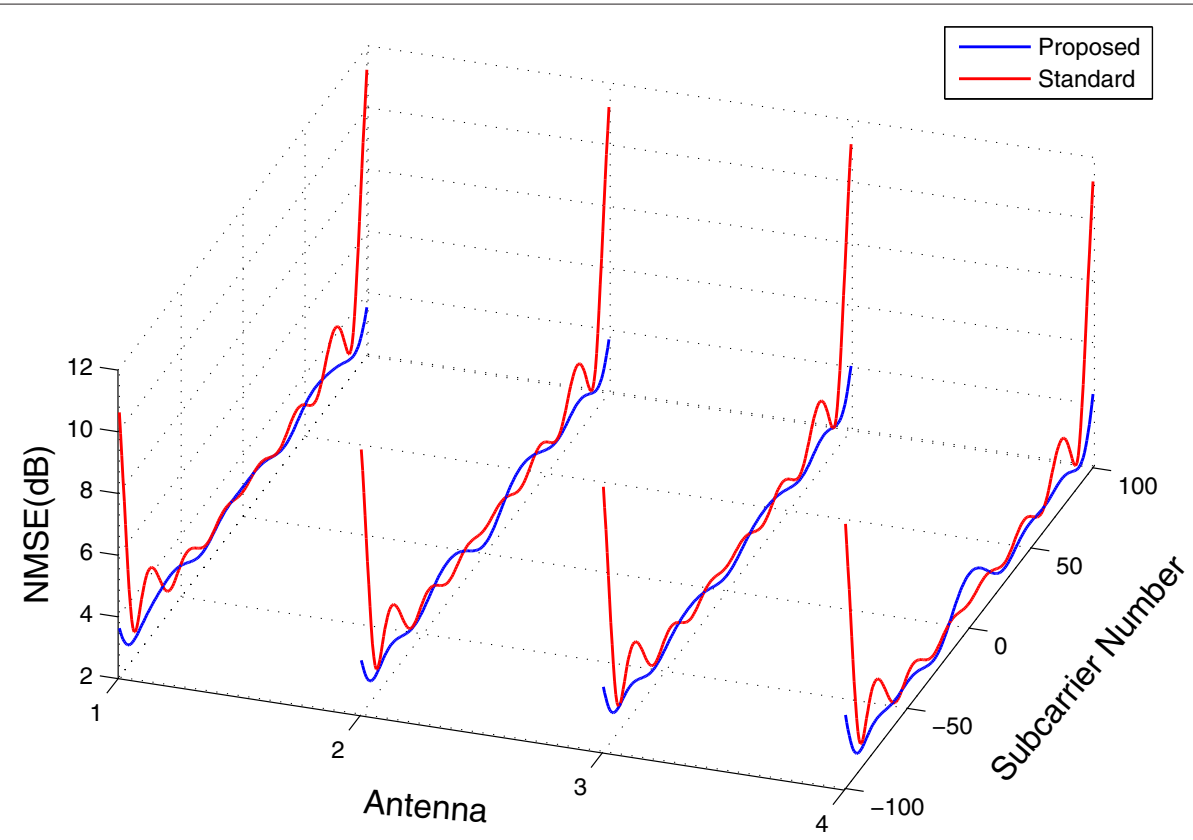

Figure 3 Comparison of channel estimate MSE between the proposed and the standard. 


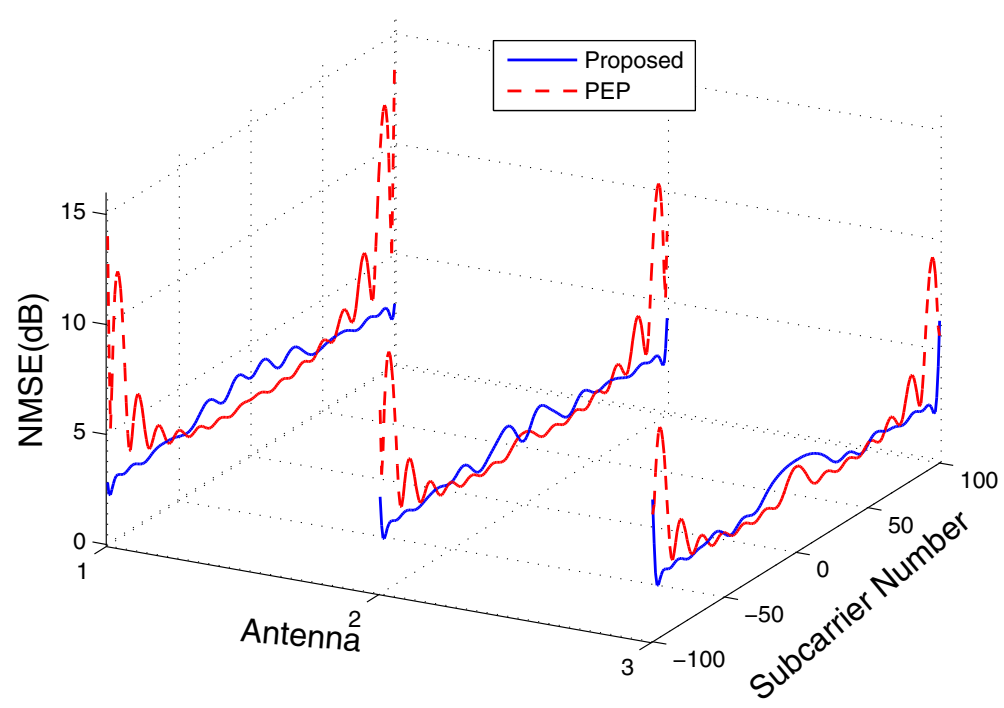

Figure 4 Comparison of channel MSE between the proposed and the PEP.

would be to increase the number of pilot subcarriers at the edge subcarriers as proposed in [33]. However, this would lower the spectral efficiency of the system. Our proposed design illuminates the improvement obtained by rearranging the pilot symbols without any addition of pilot subcarriers at the edge as suggested in [33]. This clarify that the equi-spaced and equi-powered pilot symbols are suboptimal for an OFDM system with null subcarriers.

In Figure 4, we make a comparison of the channel estimate MSE of each active subcarrier symbol for the designed disjoint pilot symbols in Figure 2. The proposed design produces MSE with small variation across all of the data subcarriers. The plot show that our proposed design outperforms the PEP for some antennas. The PEP design produces a better MSE performance for some data subcarriers around the middle of the active band. However, the design does a poor job of estimating channel at the subcarriers near the guard band. This is not due to lack of the pilot subcarriers at the edge of OFDM symbols but insignificant power allocated to the pilot symbols

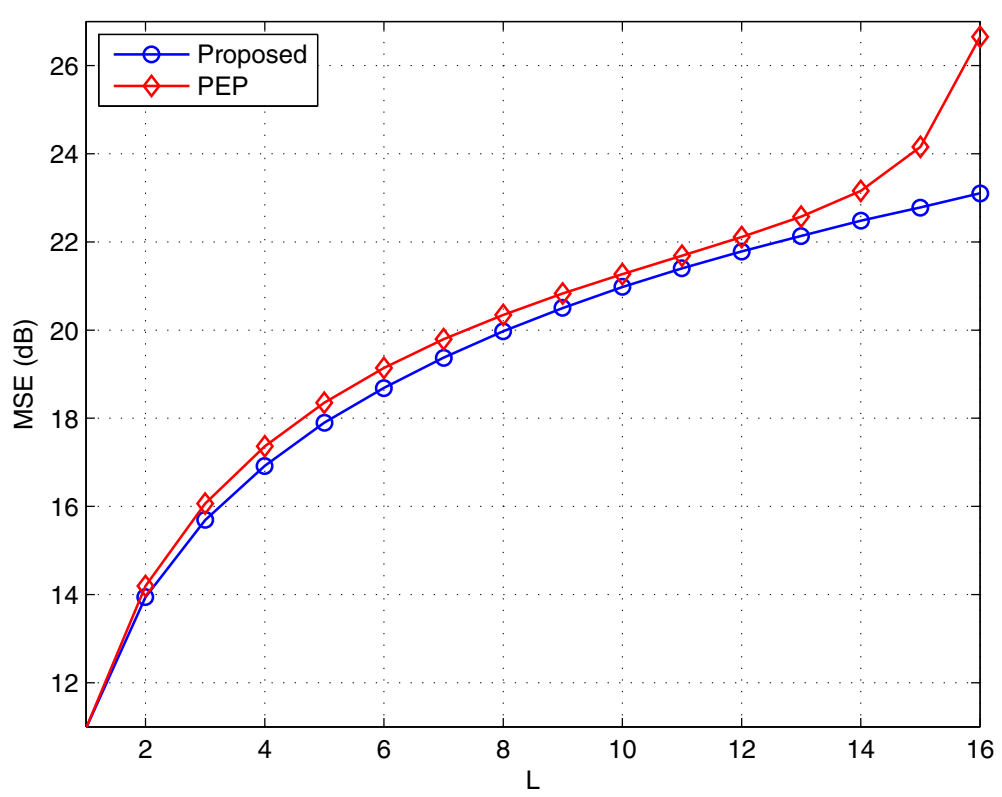

Figure 5 Channel MSE of pilot symbols for three transmit antennas. 


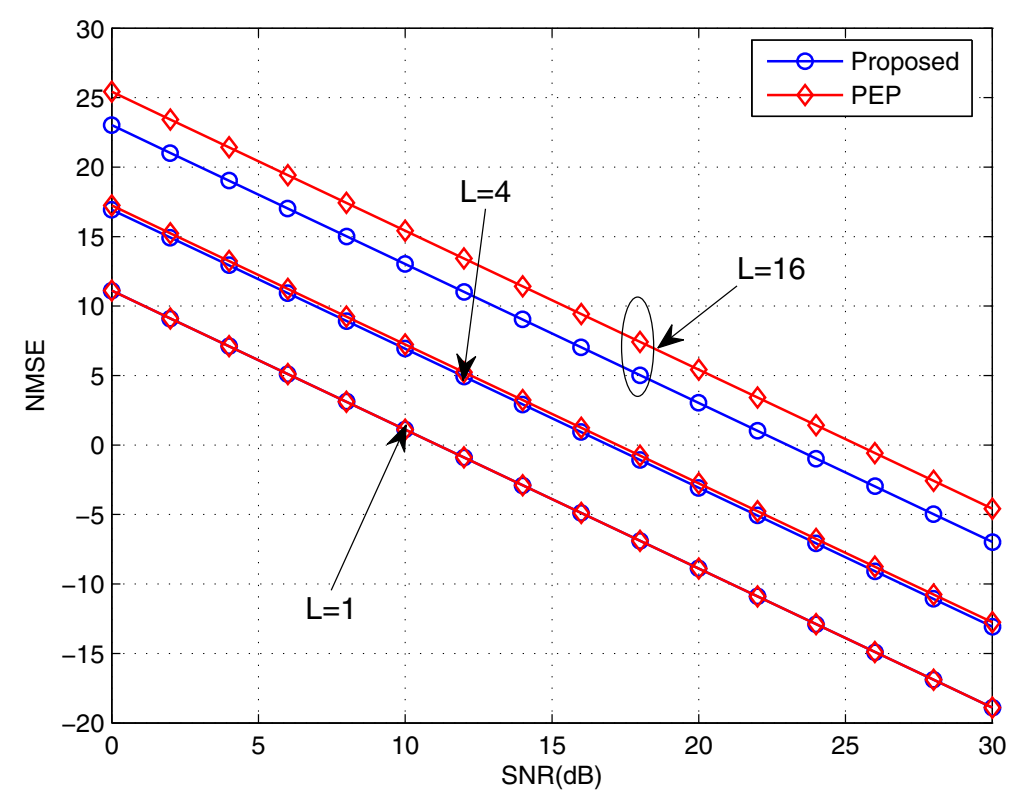

Figure 6 Comparison of the channel estimate MSE for different SNR for $N_{p}=16$ and $N_{t}=3$.

close to the null subcarrier zone. This further suggests that both pilot powers and placements need to be carefully considered in the design.

To further demonstrate the potential of our proposed design, we make a comparison of the average channel estimate MSE vs channel length $L$. To obtain the channel MSE of our proposed design as well as the PEP scheme, we varied the channel length $L$, from 1 to 16 . Figure 5 presents the average channel MSE. The proposed optimized pilot symbols exhibit better (lower) channel MSE than the PEP symbols. This clearly demonstrates the efficiency of our proposed design.

We also compare the average MSE for different signalto-noise ratio (SNR). Figure 6 depicts the average MSE versus SNR for $N_{p}=16$ for different channel length $L$. From the plot it is clear that when $L=1$ and $L=4$ the MSE performance of the PEP design is comparable to the proposed design. However, for $L=16$ the proposed

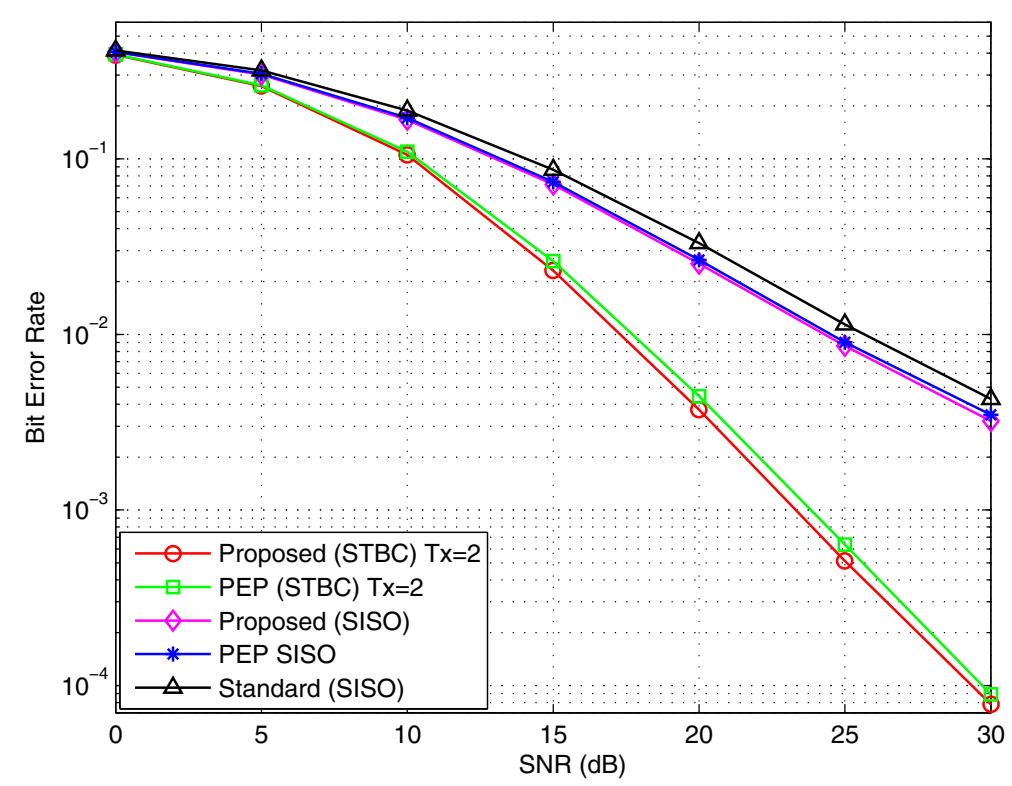

Figure 7 Comparison of BER performance for different designs for $N_{p}=8$. 


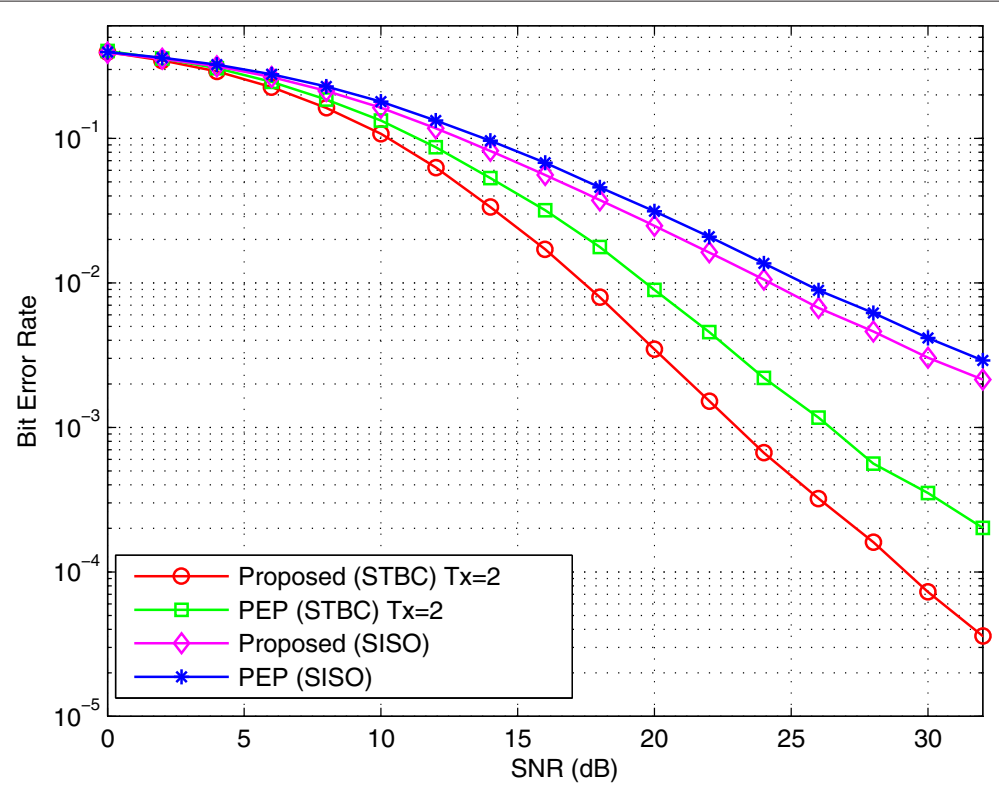

Figure 8 Comparison of the BER performance between the proposed and PEP designs for $N_{p}=16$.

design outperforms the PEP, which further corroborates the effectiveness of our proposed design over the PEP method.

\section{BER performance}

In this section, we explore the bit error rate (BER) performance gains that could be realized if the pilot symbols in IEEE $802.16 \mathrm{e}$ are designed to conform with the proposed method. We demonstrate the efficacy of our proposed method by comparing the BER performance of the proposed design, PEP and the standard pilots. The frequency selective channel with $L=N_{p}$ taps is considered. Each channel tap is i.i.d. complex Gaussian with zero mean and the exponential power delay profile is given by the vector $\rho=\left[\rho_{0} \ldots \rho_{L-1}\right]$ where $\rho_{l}=\mathcal{C} e^{-l / 2}$, and $\mathcal{C}$ is a constant selected such that $\sum_{l=0}^{L-1} \rho_{l}=1$. For MIMO case,

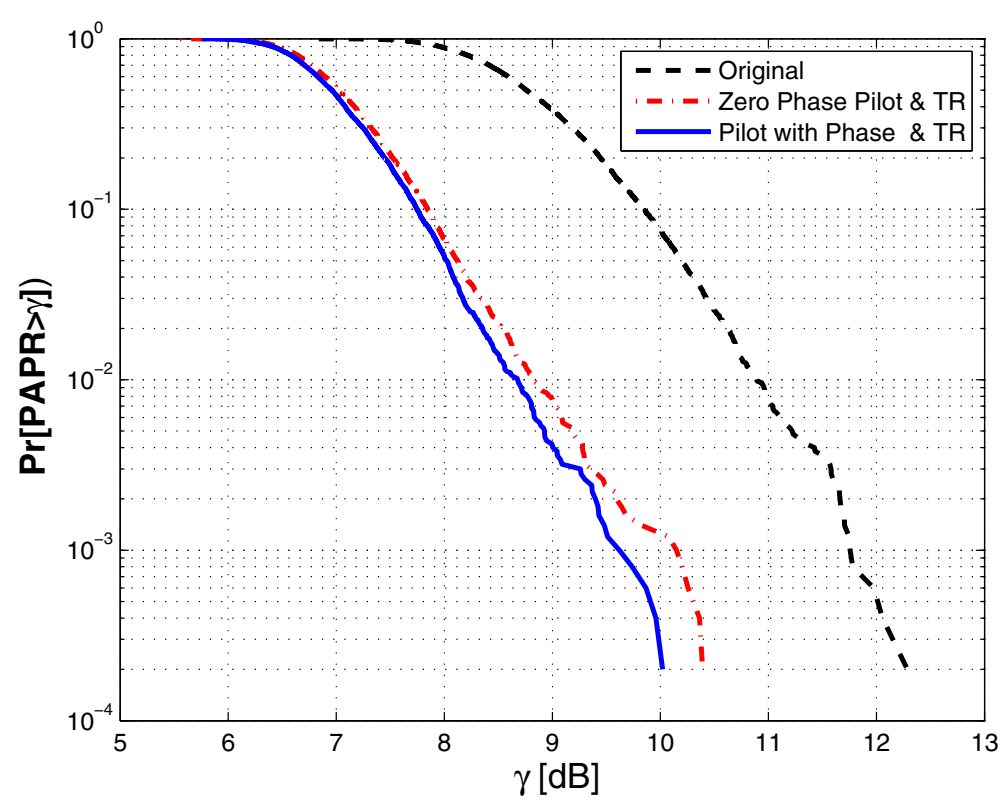

Figure 9 CCDF performance of the TR and pilot phase information for $N_{p}=16$ and $N_{t}=3$. 
to transmit an OFDM symbol, we adopt the well known Alamouti STBC for data subcarriers $\mathcal{K}_{d_{i}}$, given by $\mathcal{K}_{d_{i}} \subseteq$ $\mathcal{K}_{s} \backslash\left(\mathcal{K}_{p_{1}} \cup \mathcal{K}_{p_{2}} \ldots \cup \mathcal{K}_{p_{N_{t}}}\right)$.

Figures 7 and 8 depict the BER performance of QPSK signals for both SISO and MIMO systems. For a SISO system with $N_{p}=8$, we make a comparison of the proposed, PEP and the standard design. For the SISO case, the results in Figure 7 show identical BER performance of our proposed design and the PEP. Both PEP and the proposed design outperform the standard pilots of IEEE 802.16e. However, when two transmit antennas are considered, the proposed design provides better BER performance over the PEP design. Figure 8 verifies that, for $N_{p}=16$, the proposed design provides improved BER performance over PEP for both SISO and MIMO cases. This performance gap is a result of the PEP design having insignificant power distribution to the pilot symbols at the edge of the active subcarrier band which leads to poor estimate of the channels.

\section{PAPR designs}

To demonstrate the effectiveness of the designed TR and phase information of the pilot tones in PAPR reduction, we adopt the disjoint pilot tones in Figure 2, primarily used for channel estimations in MIMO-OFDM systems.

The PAPR CCDF of an uncoded OFDM signal are computed with an oversampling factor of $\mathcal{L}=8$ and are obtained by considering $5 \times 10^{3}$ independent OFDM symbols. The data subcarriers are loaded with QPSK signals and we consider an OFDM system with $N_{t}=3, N_{p}=16$ and the number of dummy symbols $N_{v}=16$.

Figure 9 depicts the CCDF curves of the PAPR reduction for the combined (pilot phase and TR) technique, TR technique and the original (data and pilot tones with no phase information) signals. The results show relatively lower PAPR for the OFDM symbols that utilize dummy symbols to reduce the PAPR over the data and pilot tones without any modifications. By combining phase information of the pilot tones with dummy symbols, the PAPR can further be reduced. From the figure it is clear that, there is slight improvement in PAPR reduction for the combined TR and phase information as compared to the TR technique only. This verifies the potential of our proposed combined technique in minimizing the PAPR. It should be noted that, this small improvement in PAPR due to pilot phase information is attained without any addition of resources. The total power allocated to the pilot symbols in each transmitter link is taken to be equals $N_{p}$. For system that loads more power to the pilot symbols, the significant improvement in PAPR due to phase information can be revealed. The proposed position of the dummy symbol is not necessarily optimal. Our method can adopt any available optimal location and optimize the power and phase of the dummy symbols to obtain the best possible performance. Addition of the number of dummy symbols may lead to a lower PAPR, but this will increase the total transmit power per OFDM symbol.

\section{Conclusion}

In this article, we addressed the problem of channel estimation of MIMO-OFDM systems with null subcarriers. Specifically, we extended the optimization method for designing pilot symbols in a SISO-OFDM system in [11] to MIMO systems. Through numerical simulations, we have verified that the designed pilot subcarrier set for each transmit antenna has a better channel estimate performance than the existing equi-spaced and equi-powered IEEE 802.16e standard pilot symbols and the partially equi-spaced pilot symbols. The results verified that the proposed algorithm is a prominent candidate for the design of disjoint pilot sequences for systems that utilize multiple transmit antennas and provide superior BER performance over the partially equi-spaced pilot symbols.

We also demonstrated a distortionless technique that combines TR and phase information of the pilot symbols to reducing the PAPR of the MIMO-OFDM systems. We verified that pilot symbols that are employed in wireless OFDM systems for channel estimation purpose can be extended to perform PAPR reduction as well. By combining TR and phase information of the pilot symbols, the PAPR of the time domain OFDM symbol can be significantly minimized. Through numerical simulations, we corroborated the improvement in PAPR reduction between the combined TR and pilot phase information over TR techniques only.

\section{Methods}

The analysis in this paper is conducted by using convex optimization packages (CVX) and cross entropy techniques in a MATLAB software environment to verify the theoretical expressions.

\section{Competing interests}

The authors declare that they have no competing interests.

Received: 25 October 2011 Accepted: 21 June 2012

Published: 21 June 2012

\section{References}

1. MK Ozdemir, H Arslan, Channel estimation for wireless OFDM systems. IEEE Commun. Surv. Tutor. 9(2), 18-48 (2007)

2. M Sandell, J Coon, Near-optimal training sequences for MIMO OFDM systems with nulled subcarriers. in Proc. IEEE Global Telecommunications Conference GLOBECOM '05, vol. 4, 2243-2249, (2005)

3. Q Huang, M Ghogho, S Freear, Pilot design for MIMO OFDM systems with virtual carriers. IEEE Trans. Signal Process. 57(5), 2024-2029 (2009)

4. B Rihawi, Y Louet, S Zabre, PAPR reduction scheme with SOCP for MIMO-OFDM. in Proc. Int. Conf. Wireless Communications, Networking and Mobile Computing WiCom 2007, 271-274, (2007)

5. IM Mahafeno, Y Louet, JF Helard, Peak-to-average power ratio reduction using second order cone programming based tone reservation for terrestrial digital video broadcasting systems. IET Commun. $3(7)$, 1250-1261 (2009) 
6. KC Chen, KA Morris, MA Beach, Increasing the power efficiency of an IEEE 802.11a power amplifier. in IEEE 61st Proc. VTC 2005-Spring Vehicular Technology Conference 2005, vol. 2, 954-957, (2005)

7. L Tong, BM Sadler, M Dong, Pilot-assisted wireless transmissions: general model, design criteria, and signal processing. IEEE Signal Process. Mag. 21(6), 12-25 (2004)

8. R Negi, J Cioffi, Pilot tone selection for channel estimation in a mobile OFDM system. IEEE Trans. Consum. Electron. 44, 1122-1128 (1998)

9. SOhno, G Giannakis, Optimal training and Redundant Precoding for block transmission with application to wireless OFDM. IEEE Trans. Commun. 50(12), 2113-2123 (2002)

10. S Adireddy, L Tong, H Viswanathan, Optimal placement of training for frequency-selective block-fading channels. IEEE Trans. Inf. Theory. 48(8), 2338-2353 (2002)

11. S Ohno, E Manasseh, M Nakamoto, Preamble and pilot symbol design for channel estimation in OFDM systems with null subcarriers. EURASIP $J$. Wirel. Commun. Netw. 2011, 1-17 (2011)

12. RJ Baxley, JE Kleider, GT Zhou, Pilot design for OFDM with null edge subcarriers. IEEE Trans. Wirel. Commun. 8, 396-405 (2009)

13. BR Hamilton, X Ma, JE Kleider, RJ Baxley, OFDM pilot design for channel estimation with null edge subcarriers. IEEE Trans. Wirel. Commun. 10(10), 3145-3150 (2011)

14. S Ohno, E Manasseh, M Nakamoto, Pilot symbol design for channel estimation in OFDM with null subcarriers. in Asia Pacific Signal and Information Processing Association (APSIPA), 696-699, (2009)

15. S Myeongchoel, L Hakju, L Chungyong, Enhanced channel-estimation technique for MIMO-OFDM systems. IEEE Trans. Veh. Technol. 53, 261-265 (2004)

16. EG Larsson, J Li, Preamble design for multiple antenna OFDM-based WLANs with null subcarriers. IEEE Signal Process. Lett. 8(11), 325-327 (2001)

17. Z Li, Y Cai, Y Xu, Optimal training signals design for MIMO OFDM systems with guard subcarriers. in IEEE 63rd Proc. VTC 2006-Spring Vehicular Technology Conference, vol. 3, 1541-1544, (2006)

18. A Dowler, A Nix, Performance evaluation of channel estimation techniques in a multiple antenna OFDM system. in IEEE 58th Proc. VTC 2003-Fall Vehicular Technology Conf. 2003, vol. 2, 1214-1218, (2003)

19. HD Tuan, HH Kha, HH Nguyen, VJ Luong, Optimized training sequences for spatially correlated MIMO-OFDM. IEEE Trans. Wirel. Commun. 9(9), 2768-2778 (2010)

20. RFH Fischer, M Hoch, Peak-to-average power ratio reduction in, MIMO OFDM. in Proc. IEEE Int. Conf. Communications ICC'07, 762-767, (2007)

21. E Manasseh, S Ohno, M Nakamoto, Design of low PAPR preamble and pilot symbol for channel estimation in OFDM systems. Int. J. of Innov. Comput. Inf. Control. 7, 39-50 (2011)

22. S Hosokawa, K Teo, S Ohno, T Hinamoto, Pilot tone design with low peak-to-average power ratio in OFDM. IEICE Trans. Fund. E88-A(8), 2117-2123 (2005)

23. S Janaaththanan, C Kasparis, BG Evans, A gradient based algorithm for PAPR reduction of OFDM using tone reservation technique. in Proc. IEEE Vehicular Technology Conf. VTC Spring 2008, 2977-2980, (2008)

24. D Guel, J Palicot, Y Loue, Tone reservation technique based on geometric method for orthogonal frequency division multiplexing peak-to-average power ratio reduction. IET Commun. 4(17), 2065-2073 (2010)

25. C Jung-Chieh, L Chih-Peng, Tone reservation using near-optimal peak reduction tone set selection algorithm for PAPR reduction in OFDM systems. IEEE Signal Process. Lett. 17(11), 933-936 (2010)

26. R Cavalcante, I Yamada, A flexible peak-to-average power ratio reduction scheme for OFDM systems by the adaptive projected subgradient method. IEEE Trans. Signal Process. 57(4), 1456-1468 (2009)

27. E Lawrey, CJ Kikkert, Peak to average power ratio reduction of OFDM signals using peak reduction carriers. in Proc. Fifth Int. Symp. Signal Processing and Its Applications ISSPA'99, vol. 2, 737-740, (1999)

28. A Erdogan, A low complexity multicarrier PAR reduction approach based on subgradient optimization. Signal Process. 86(12), 3890-3903 (2006)

29. A Aggarwal, TH Meng, Minimizing the peak-to-average power ratio of OFDM signals using convex optimization. IEEE. Trans. Signal Process. 54(8), 3099-3110 (2006)

30. S Boyd, L Vandenberghe, Convex Optimization. (Cambridge: Cambridge University Press, 2004)
31. IEEE: IEEE Standard for Local and Metropolitan Area Networks Part 16: Air Interface for Fixed Broadband Wireless Access Systems. IEEE 2004

32. E Manasseh, S Ohno, M Nakamoto, Pilot symbol design for channel estimation in MIMO-OFDM systems with null subcarriers. in European Signal Processing Conference, 1612-1616, (2010)

33. M Morelli, U Mengali, A comparison of pilot-aided channel estimation methods for OFDM systems. IEEE. Trans. Signal Process. 49(12), 3065-3073 (2001)

doi:10.1186/10.1186/1687-1499-2012-201

Cite this article as: Manasseh et al: Combined channel estimation and PAPR reduction technique for MIMO-OFDM systems with null subcarriers. EURASIP Journal on Wireless Communications and Networking 2012 2012:201.

\section{Submit your manuscript to a SpringerOpen ${ }^{\circ}$ journal and benefit from:}

- Convenient online submission

- Rigorous peer review

- Immediate publication on acceptance

- Open access: articles freely available online

- High visibility within the field

- Retaining the copyright to your article

Submit your next manuscript at $\boldsymbol{\wedge}$ springeropen.com 